Results We confirm the initially reported specificity and further narrow down its confidence interval (specificity 99.5\%, 95\% CI 99.4-99.6\%), and show that this high specificity is valid across diverse patient categories. Here we demonstrate that differences in positive predictive values between patient categories reflect the prevalence of syphilis in these categories, and are not due to differences in specificity. In addition, in a sensitivity analysis we show that these conclusions are robust for several assumptions.

Conclusion Our analysis shows that the high specificity found in the initial study, stands up after implementation in a population with a low syphilis prevalence $(0.9 \%)$. Using a selected serum sample collection is therefore a valid manner in the evaluation of syphilis serological diagnostic assays. Confirmatory syphilis testing remains mandatory in low prevalence populations, even when the screening test has a very high specificity.

\section{P5.090 EVALUATION OF A DOUBLE RAPID TEST FOR SYPHILIS AND HIV: SD BIOLINE HIV/SYPHILIS DUO}

doi:10.1136/sextrans-2013-051184.1134

'M A Chiappe, 'L Lopez-Torres, 'C Carcamo, 'P Garcia, ${ }^{2} \mathrm{R}$ Peeling. ' Universidad Peruana Cayetano Heredia, Lima, Peru; ${ }^{2}$ Clinical Research Unit, ITD London School of Hygiene \& Tropical Medicine, London, UK

Background Although syphilis and HIV are primarily transmitted through sexual intercourse, they can also be transmitted from mother to child during pregnancy or at delivery. Testing pregnant women for syphilis and HIV is an important public health measure to prevent vertical transmission. Several countries have included screening of pregnant women using rapid HIV testing, and recently also rapid syphilis testing. Screening pregnant women for both diseases with one test is not only desirable but could also be very convenient. We present the results of the laboratory-based evaluation of a new test: the SD Bioline HIV/Syphilis Duo.

Methods We used archived serum specimens characterised as positive or negative for HIV and/or Syphilis. The gold standard positive for HIV was EIA (Vironostika ${ }^{\circledR}$ HIV Uni-Form II Ag/Ab, bioMérieux) with confirmation using Western blot. The reference standard positive for syphilis was the Rapid Plasma Reagin test (RPR, bioMérieux) with confirmation using the Treponema pallidum Particle Agglutination (TPPA) assay (Fujireibio, Japan). Reference standard negatives were EIA negative and RPR negative for HIV and syphilis respectively. For Syphilis we used a total of 665 samples, including 198 positives, and for HIV we used 662 samples including 91 positives. There were 42 samples positive for both HIV and syphilis. The samples were tested with the SD Bioline HIV/Syphilis Duo by a laboratory technician blinded to the gold standard results.

Results For Syphilis we observed a sensitivity of 100\% (198/198) and a specificity of $99.57 \%$ (465/467). The two RPR negative/Bioline positive samples were negative for TPPA. For HIV, both the sensitivity and specificity were 100\% (91/91 and 571/571 respectively). Conclusions The SD Bioline HIV/Syphilis Duo test has a good performance in archived sera. Its high sensitivity suggests that this dual test would be of use in screening programmes for syphilis and HIV.

\section{P5.091 HEAD-HEAD COMPARISON OF REACTIVITY AND SIGNAL STRENGTH VALUE FOR REACTIVITY AMONG SEVEN TREPONEMAL ASSAYS: A PRELIMINARY REPORT}

doi:10.1136/sextrans-2013-051184.1135

'Y F Fakile, ' $\mathrm{H}$ Jost, 'S Kikkert, ' $\mathrm{K}$ W Hoover, ${ }^{2} \mathrm{~J} \mathrm{M}$ Schapiro, ${ }^{3} \mathrm{~S}$ M Novak-Weekley, ${ }^{4} \mathrm{~J}$ M Chow, ${ }^{4} \mathrm{U}$ Park. 'Centers for Disease Control and Prevention, Atlanta, GA, United States, ${ }^{2}$ Regional Laboratory, Kaiser Permanente Northern California, Berkeley, CA, United States, ${ }^{3}$ SCPMG Regional Reference Laboratories, North Hollywood, CA, United States, ${ }^{4}$ CA Dept of Public Health-STD Control Branch, Richmond, CA, United States
Background Automated immunoassays (AI) for detection of $T$. pallidum antibodies are increasingly used for syphilis screening in the United States. These assays demonstrate fast performance, reduced labour requirements, and high throughput with walk-away capability. Limited data are available about the relative seroreactivity among commercial treponemal assays, especially in low risk populations. Additionally, it is unknown to what extent the AI signal strength values, used to assess reactivity, are associated with non-AI treponemal reactivity. We compared concordance of seroreactivity among 7 treponemal tests and assessed AI signal strength values associated with reactivity.

Methods Previously identified reactive and nonreactive sera $(n=566)$ were obtained from Kaiser Northern and Southern California regional laboratories. All sera were tested with AIs: BioPlex 2200 Syphilis IgM/IgG (BioRad), treponemal LIAISON (DiaSorin), Advia-Centaur syphilis (Siemens), and non-AIs (INNOLIA syphilis score (INNOGENETICS), TrepSure (Phoenix Biotech), Treponemal Pallidum Particle Agglutination (TP-PA) (Fujirebio), and Fluorescent Treponemal Antibody-Absorption (FTA-ABS) (Zeus Scientific) tests. Reactivity was interpreted according to manufacturers' instructions.

Results Seroreactivity ranged from $40.5-43.9 \%$ for AIs, and 33.0 $42.2 \%$ for non-AIs. In all 7 tests, 30\% (167/566) were reactive, and positive agreement among assays was $82.3 \%$. The overall seroreactivity among AIs was $38.9 \%(220 / 566)$ and positive agreement was $92.6 \%$. Minimum signal strength values of 11.72 (Centaur, range: 1.1-45), 4.4 (BioPlex, range: 1.1-8) and 9.4 (Liaison, range: 1.1-70) correlated $100 \%$ with TPPA reactivity. The proportion of AI-seroreactive specimens that were also TP-PA reactive were: $86.5 \%$ $(198 / 229)$ for BioPlex, 85.2\% (202/237) for ADVIA-Centaur, and $81.6 \%(200 / 245)$ for LIAISON.

Conclusion Although there is some variation in seroreactivity among the 7 tests, there is good correlation. A large proportion of $\mathrm{AI}$ tests with a minimal signal-to-cutoff ratio were associated with a positive TP-PA, suggesting that a second treponemal test may not be necessary to confirm AI-reactive, RPR-nonreactive sera.

\section{P5.092 EVALUATION OF A LABORATORY DEVELOPED TEST FOR THE DETECTION OF TRICHOMONAS VAGINALIS USING A MODIFICATION OF THE ABBOTT M2000 REALTIME SYSTEM}

doi:10.1136/sextrans-2013-051184.1136

'J A Williams, 'L Eddleman, 'A LeMonte, 1'2B Van Der Pol. 'Indiana University School of Medicine, Indianapolis, IN, United States; ${ }^{2}$ Indiana University School of Public Health, Bloomington, IN, United States

Background Assays for the detection of Trichomonas vaginalis (TV) are available on certain commercial platforms. The objective of this study was to assess the performance characteristics of a new laboratory developed test (LDT) for the detection of TV from urine, and swab samples, when tested on the Abbott $m 2000$ platform; a platform widely used for the detection of $C$. trachomatis (CT) and $N$. gonorrhoeae (NG).

Methods Residual swab samples that had been previously eluted into CT transport medium and urine were placed into Abbott transport tubes. Testing for CT/NG was performed on the $m 2000$ platform per package insert; the remaining residual extracted DNA was used for TV testing on the $m 2000$ platform. TV specific primers, probe, and thermal cycling conditions were optimised in our laboratory. Residual DNA from each sample was manually transferred to an amplification plate containing master mix. Real-time PCR was performed on the m2000 platform in open mode with the TV LDT results being compared to an LDT for TV that has been validated and used in our laboratory for more than a decade. Assay agreement was assessed using Kappa statistics. 